\title{
Strain selection of charge and orbital ordering patterns in half-doped manganites
}

\author{
M.J. Calderón \\ Cavendish Laboratory, Cambridge University, Madingley Road, Cambridge CB3 OHE, UK \\ A.J. Millis \\ Department of Physics, Columbia University, 538 W. 120th St, New York, NY 10027 \\ K.H. Ahn \\ Theoretical Division, Los Alamos National Laboratory, Los Alamos, New Mexico 87545
}

(Dated: June 4, 2018)

\begin{abstract}
Theoretical and computational results are presented clarifying the role of long-ranged strain interactions in determining the charge and orbital ordering in colossal magnetoresistance manganites. The strain energy contribution is found to be of order $20-30 \mathrm{meV} / \mathrm{Mn}$ and in particular stabilizes the anomalous 'zig-zag chain' order observed in many half-doped manganites.
\end{abstract}

Perovskite manganites of chemical formula $\mathrm{Ln}_{1-x} \mathrm{Ak}_{x} \mathrm{MnO}_{3}$ ( $\mathrm{Ln}$ is a lanthanide rare earth such as $\mathrm{La}$ or $\mathrm{Pr}$; $\mathrm{Ak}$ is a divalent alkali such as $\mathrm{Sr}$ or $\mathrm{Ca}$ ) present a complex phase diagram associated with the interplay of charge, spin, orbital and lattice degrees of freedom 1 Charge and orbitally ordered phases appear in wide parameter ranges, for example in $\mathrm{La}_{1-x} \mathrm{Ca}_{x} \mathrm{MnO}_{3}$ for $x \geq 0.5$, and in $\mathrm{Pr}_{1-x} \mathrm{Ca}_{x} \mathrm{MnO}_{3}$ for $0.3 \leq x \leq 0.7$. The physical mechanism underlying the ordering remains the subject of debate. Particular attention has focussed on the half-doped $(x=0.5)$ materials, including $\mathrm{La}_{0.5} \mathrm{Ca}_{0.5} \mathrm{MnO}_{3}, \mathrm{Pr}_{0.5} \mathrm{Ca}_{0.5} \mathrm{MnO}_{3}$ and $\mathrm{Nd}_{0.5} \mathrm{Sr}_{0.5} \mathrm{MnO}_{3}$, which possess a strongly insulating ground state with a particularly interesting ('zig-zag chain') spin, charge and orbital ordering pattern shown in the inset to Fig. 11. Stabilizing this state requires 'second neighbor' interactions, which as noted by Khomskii and Kugel ${ }^{2}$ and by Ahn and Millis ${ }^{3}$ may reasonably be expected to arise from electron-lattice interactions. However, experiments have also indicated that long ranged strain plays an important role. Insulating ordered phases appearing in bulk $\mathrm{La}_{0.5} \mathrm{Ca}_{0.5} \mathrm{MnO}_{3}$ are suppressed in thin films ${ }^{4}$; it is argued that the difference arises from the lattice mismatch with the substrate, which prevents the occurrence of lattice distortions necessary to accommodate orbital ordering. Similar conclusions have been drawn from recent work on $\mathrm{Pr}_{0.6} \mathrm{Ca}_{0.4} \mathrm{MnO}_{3}$ films subjected both to compressive and tensile strain 5 Other sets of experiments in polycrystalline $\mathrm{La}_{0.5} \mathrm{Ca}_{0.5} \mathrm{MnO}_{3}$ show that the charge/orbital ordering transition temperature is progressively suppressed as grain size is decreased; the interpretation again is that boundary effects inhibit the formation, in small grains, of the strains imposed by the orbital ordering. 6 Perhaps most significantly, polarized optical microscopy studies on $\mathrm{Bi}_{0.2} \mathrm{Ca}_{0.8} \mathrm{MnO}_{3}$ single crystals 7 reveal that as temperature is decreased to just below the charge/orbital ordering transition, lenticular shaped domains appear and grow slowly with time. This behavior is well known in martensitic systems ${ }^{8}$, where it is attributed to the interplay between a tendency of the system to favor a state with large strain, and a boundary condition which prevents the existence of a truly uniform strain.

Despite the considerable experimental evidence that long ranged strain is important, strain physics has not received much theoretical attention. The original analyses 9.10 .11 and many subsequent works ${ }^{12.13 .14}$ focused on local interactions, including magnetic, Coulomb and Jahn-Teller electron-phonon coupling. In this paper we study long ranged strain effects theoretically, and in particular show that they play a crucial role in stabilizing the particular ordered state characteristic of many of the half doped materials.

The $\mathrm{Ln}_{1-x} \mathrm{Ak}_{x} \mathrm{MnO}_{3}$ materials have approximately the cubic perovskite crystal structure, in which the Mn ions sit on the vertices of a simple cubic lattice. The important low-energy electronic degrees of freedom may be thought of as Mn $e_{g}$ electrons. There are two $e_{g}$ orbitals per Mn. These are degenerate in cubic symmetry. The $(1-x) e_{g}$ electrons are relatively mobile at room temperature but in many manganite materials, including the half-doped compounds of present interest, they become strongly localized at low temperatures. The $x \neq 0$ insulating states have a charge density which varies from Mn to Mn; the pattern of charge density is referred to as 'charge order'. In many of the half-doped materials, periodic charge order is observed, with wave vector $(\pi, \pi, 0)$ : in other words, in a given plane (which we take to be the $x y$ plane) of the ideal cubic perovskite structure the charge alternates from site to site in a simple two sublattice fashion, but the charge ordering simply stacks uniformly in the perpendicular direction. The observation of $(\pi, \pi, 0)$ is surprising: charge ordering typically occurs because electrons repel each other, and a fully alternating $(\pi, \pi, \pi)$ ordering is typically preferred because it allows the greatest distance between charges.

If charges are localized, then one expects that on a given site one linear combination of the two $e_{g}$ orbitals will have a higher occupancy than the orthogonal linear combination. The pattern of this preferential occupancy is referred to as 'orbital ordering'. It was first inferred from the magnetic ordering pattern (discussed be- 
low) by Wollan and Koehler in the 1950s 15 using rules determined by Goodenough ${ }^{9}$ and Kanamori ${ }^{10}$, and has now been studied in numerous samples by resonant $\mathrm{x}$-ray scattering 16 , which is sensitive both to the orbital occupancy and to the changes it induces in oxygen positions. The orbital ordering occurring in the strongly insulating half doped materials may be visualized as 'zig-zag chains' on the $x y$-plane stacked uniformly in the $z$-direction. The 'zig-zag' is produced by the alternation of preferred occupancy of $3 x^{2}-r^{2}$ and $3 y^{2}-r^{2}$ orbitals on the sublattice of sites with high charge occupancy (see inset to Fig. 1 $\mathrm{l}$ ). The magnetic ordering pattern corresponds to ferromagnetism along the 'zig-zag chains' but with spin direction alternating from chain to chain, and follows from the application of the Goodenough/Kanamori rules to the zigzag pattern of states 11 . We note, however, that strain physics should also be important because the $e_{g}$ orbitals have a non-cubic shape. Thus, in the case of 'zig-zag' ordering, the occupied orbitals have charge density lying mainly in the 'xy' plane, leading to a compression in the perpendicular direction.

We now turn to the theory. A general model would include electronic dynamics (hopping and electron-electron interaction), electron-lattice coupling, and the energetics associated with the lattice degrees of freedom. Because we wish to focus on the new physics associated with strain effects, and because the main application we have in mind is to strongly insulating half-doped manganites, for simplicity we adopt a classical, insulating model in which the electrons are taken to be localized on Mn sites, but our approach may easily be extended to an itinerant electron model. In the insulating model the $\mathrm{e}_{g}$ orbitals of a Mn site can be either empty, or occupied by one electron which resides in a linear combination of the two $e_{g}$ orbitals. We parameterize the charge state of site $i$ by a variable $h_{i}$ with $h_{i}=0$ for an occupied site and $h_{i}=1$ for an empty site, so doping $x=\left(\sum_{i} h_{i}\right) / N$ and we focus here on $x=0.5$. The orbital state of an occupied site is described by an angle $0 \leq$ $\theta_{i} \leq \pi$ so that $\left|\psi_{i}\left(\theta_{i}\right)\right\rangle=\cos \theta_{i}\left|d_{3 z^{2}-r^{2}}\right\rangle+\sin \theta_{i}\left|d_{x^{2}-y^{2}}\right\rangle$. (Note that $\left|d_{3 z^{2}-r^{2}}\right\rangle,\left|d_{3 x^{2}-r^{2}}\right\rangle,\left|d_{3 y^{2}-r^{2}}\right\rangle$ correspond to $\theta_{i}=0, \pi / 3,2 \pi / 3$ respectively while $\left|d_{z^{2}-x^{2}}\right\rangle,\left|d_{x^{2}-y^{2}}\right\rangle$, $\left|d_{z^{2}-y^{2}}\right\rangle$, correspond to $\theta_{i}=\pi / 6, \pi / 2,5 \pi / 6$.)

The electron-lattice coupling is taken to have the form given in Ref. [13]: a site containing an electron is JahnTeller coupled to the surrounding oxygen octahedron and an empty site induces a breathing distortion of the octahedron. In an isolated $\mathrm{MnO}_{6}$ octahedron the JahnTeller effect would lead to an energy gain $E_{J T}=\lambda^{2} / 2 K_{1}$ with $\lambda$ the Jahn-Teller coupling and $K_{1}$ the Mn-O bondstretching force constant (see the inset to Fig. 10). Optical measurements on $\mathrm{LaMnO}_{3}$ suggest $K_{1} \approx 7.36 \mathrm{eV} / \AA^{2}$ and both band structure calculations and fitting to the observed structure suggest $\lambda \approx 1.38 \mathrm{eV} / \AA$ so that $E_{J T} \approx$ $0.26 \mathrm{eV}$. We adopt this as the basic energy scale of the theory. We write the energy gain due to a breathing distortion as $E_{B}=\beta E_{J T}$. We expect that the energy costs of the two distortions are similar, so $\beta \sim 1$.
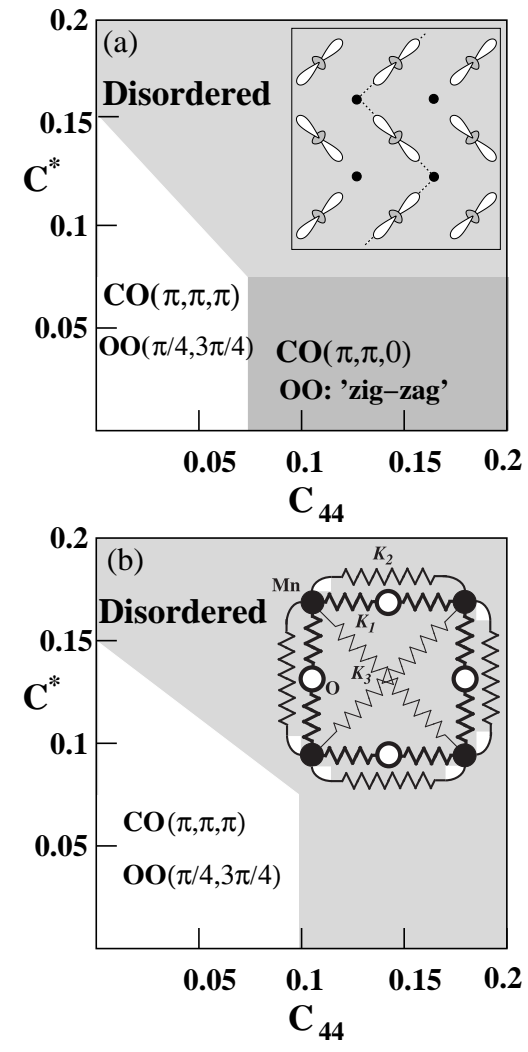

FIG. 1: Main panels: ground-state phase diagrams calculated by Monte-Carlo annealing on $8 \times 8 \times 8$ lattices, with (a) and without (b) the inclusion of the long range energy. The wave vector of the charge order $(\mathrm{CO})$ and the pattern $\theta_{i}$ of the orbital ordering (OO) are indicated. 'Disordered' corresponds to phases which show no obvious periodicity or symmetry on the lattices studied. Calculations are performed for $\beta=0.8$ for various force constants consistent with bulk modulus $C_{B}=$ $0.35 K_{1}$ and are presented as functions of the shear $C_{44}$ and Jahn-Teller $C^{*}$ elastic moduli implied by the force constants and expressed in units of $K_{1}$. The inset to Fig. 10 shows the 'zig-zag' pattern. Black dots represent the sites with low charge occupancy. (b)The 'zig-zag' pattern is absent when uniform strain energy is neglected. The inset to Fig. 10 shows three of the force constant model couplings. Additional noncentral force coupling to Mn-Mn-Mn bond angles not shown.

In the actual perovskite lattice, each $\mathrm{O}$ ion is bonded strongly to two Mn, so a breathing or Jahn-Teller distortion about one site is coupled to distortions at neighboring sites. To incorporate this physics we follow Refs. 13,17] and write a model whose variables are the deviations $\vec{\delta}_{i}$ of the Mn ions from their ideal cubic perovskite positions and the displacements $\vec{u}_{i}$ of the O-ion at $\vec{i}+\hat{a} / 2$ along $\hat{a}$ direction $(a=x, y, z)$. These variables are coupled harmonically by near neighbor force constants. The inset to Fig. 1b shows the planar force constants. We parameterize these force constants by the bulk $\left(C_{B}\right)$, cubictetragonal $\left(C^{*}\right)$ and shear $\left(C_{44}\right)$ moduli which they imply. We then integrate out the variables describing the 
O positions and obtain an energy of the form

$$
E_{\text {tot }}\left(\left\{h_{i}, \theta_{i}, \delta_{i}\right\}\right)=E_{\text {lattice }}\left(\left\{\delta_{i}\right\}\right)+E_{\text {JT, breath }}\left(\left\{h_{i}, \theta_{i}, \delta_{i}\right\}\right)
$$

(A detailed derivation will be given in a separate communication $\left.{ }^{18}\right)$.

For each electronic configuration $\left(\left\{h_{i}, \theta_{i}\right\}\right)$ we may now obtain the total energy by minimizing Eq. 1 with respect to the lattice variables $\vec{\delta}_{i}$. We have used a classical Monte Carlo simulation process on $4 \times 4 \times 4$ and $8 \times 8 \times 8 \mathrm{Mn}$ lattices to find the ground state by annealing the system starting from a random high temperature configuration. However, an issue arises in the evaluation of the energy. To describe this, note that the term $E_{\mathrm{JT} \text {,breath }}$ may be conveniently written in momentum space as a linear coupling between the Fourier transform of the displacements, $\vec{\delta}_{k}$, and a vector $\vec{e}_{k}$ constructed from the $h_{i}, \theta_{i}$ and the parameter $\beta: E_{\mathrm{JT}, \text { breath }}=\sum_{k} \vec{e}_{-k} \boldsymbol{\Lambda}(k) \vec{\delta}_{k}$. The purely lattice term may similarly be written as $E_{\text {lattice }}=\frac{1}{2} \sum_{k} \vec{\delta}_{-k} \mathbf{K}(k) \vec{\delta}_{k}$ with $\mathbf{K}$ a force-constant matrix, so that after minimization over the $\delta$ we have

$$
E_{\mathrm{tot}}=-\frac{1}{2} \sum_{k} \vec{e}_{-k}\left[\boldsymbol{\Lambda}(k) \mathbf{K}^{-1}(k) \boldsymbol{\Lambda}(k)\right] \vec{e}_{k}
$$

On a finite lattice the sum is over a discrete set of $k$ points and the terms with $k \neq 0$ may straightforwardly be evaluated. However, as $k \rightarrow 0$ we find $\boldsymbol{\Lambda}(k) \sim k$ and $\mathbf{K}(k) \sim k^{2}$ so that the $\lim _{k \rightarrow 0}\left[\boldsymbol{\Lambda}(k) \mathbf{K}^{-1}(k) \boldsymbol{\Lambda}(k)\right] \sim \frac{0}{0}$. The limit, and therefore the value of the $k=0$ term in Eq. 2 depends on the direction from which $k$ approaches 0 . These different limits correspond to different uniform strain states of the material (and therefore to different changes to shape of the whole lattice), and require special attention. We find that for almost every configuration of $h_{i}, \theta_{i}$ there is one particular strain state (direction along which the $k \rightarrow 0$ limit is taken) which minimizes $E_{t o t}$. We use two annealing procedures. In one, we use the $E_{\text {tot }}$ corresponding to this 'optimum' strain state, thereby updating the strain every time the electronic configuration is changed and allowing the system to minimize its energy with respect to strain ${ }^{19}$ In the other, we neglect the $k=0$ term in Eq. 2] entirely. One could also constrain the $k=0$ Fourier components of $\delta_{i}$ to take particular values, thereby forcing the system to accommodate to a definite strain (to simulate, e.g. a film grown on a lattice-mismatched substrate), but we do not explore this physics here.

We have conducted extensive simulations of Eq. 1 for different parameters. The bulk modulus has been estimated to be $135 \mathrm{GPa}\left(0.35 \text { in units of } \mathrm{K}_{1}\right)^{20}$ and we consider only choices of $K_{i}$ consistent with this value. We present our results (obtained by varying the $K_{i}$ in a manner consistent with the experimental bulk modulus) in terms of the value of the other elastic moduli. Different values for $\beta$ have been explored, but here we only show results for $\beta=0.8$.

Fig. 1) shows typical ground-state phase diagrams arising from our simulations. The main panel in Fig. 17 shows the phase diagram following from optimizing the full model over strain. Three states are observed: one $(C O=(\pi, \pi, \pi) ; O O=(\pi / 4,3 \pi / 4))$ corresponds to the arrangement of charges and orbitals which implies the lowest uniform strain; another, labeled 'Disordered' in the figure, corresponds to states which have no obvious ordering pattern within the $8 \times 8 \times 8$ unit cells we can access numerically, and which presumably either have some extremely long-ranged periodicity or are glassy. We note that in this parameter regime these 'disordered' states are found by the annealing procedure to have energies lower than those of any simple periodic structure we have devised.

In the lower right region of the phase diagram in Fig. 17 the preferred state corresponds to the one found in experiments. Because it involves quite substantial anisotropic lattice distortions it is stabilized only in particular regions of the phase diagram. The main panel of Fig. 10 shows the results of computations for the same parameters, but with the uniform strain $(k=0)$ contribution to the energy neglected. We see that the experimentally observed phase is entirely absent. This conclusion remains true in the range $0.6 \leq \beta \leq 1.2$. Details on the role of $\beta$ will be given in a separate communication 18

The difference in phase diagrams computed with and without the strain contribution to the energy suggests the importance of long ranged strain effects. To understand this result in more detail we have evaluated both the long range $(k=0)$ and short range $(k \neq 0)$ contributions to $E_{t o t}$ for many different orbital orders, and $(\pi, \pi, 0)$ and $(\pi, \pi, \pi)$ charge order. Typical results are presented in Table I. One sees that the differences in strain energy between different orbitally ordered phases $(\sim 20 \mathrm{meV} / \mathrm{Mn})$ are of the same order as magnetic energy differences computed by other workers 14 . Observe that the uniform strain energy only depends on the orbital configuration, and is optimized for orbitals which correspond to mainly planar charge densities. The 'zigzag' pattern of orbitals is consistent with this result. The 'uniform clover' pattern (all orbitals $\theta=\pi / 2$ i.e. $d_{x^{2}-y^{2}}$ ) has an even lower strain energy, but in the insulating model considered here its short range energy is never large enough to stabilize this phase. We note, however, that electron banding effects are found theoretically to favor this phase $\mathrm{e}^{21}$ and that it is observed in metallic $\mathrm{La}_{0.5} \mathrm{Sr}_{0.5} \mathrm{MnO}_{3}$. On the contrary, the $(\pi / 4,3 \pi / 4)$ orbital state is the least strained configuration of all the ordered ones, the reason for this being its isotropic charge distribution. Note also that the strain term does not distinguish between different $\mathrm{z}$-direction orderings: it is the short range part of the energy which chooses the stacking of charge in the z-direction $((\pi, \pi, 0)$ vs $(\pi, \pi, \pi))$.

We now comment on possible extensions of our model. Magnetic interactions have been neglected, but could be easily included. The $t_{2 g}$ localized spins are antiferromagnetically coupled via superexchange. There is also a ferromagnetic superexchange interaction between the $e_{g}$ spins which depends on the occupied orbitals in two 
TABLE I: Energies for different orbital (OO) and charge ordering (CO) patterns as indicated, calculated for parameters $\beta=0.8$, $C_{B}=135 \mathrm{GPa}\left(0.35 K_{1}\right), C^{*}=17.35 \mathrm{GPa}\left(0.045 K_{1}\right)$, and $C_{44}=30.85 \mathrm{GPa}\left(0.08 K_{1}\right)$ chosen within the range in which the 'zig-zag' ordering is the ground state (see Fig 11).

\begin{tabular}{c|c|c|c}
\hline & Uniform Strain Energy $(\mathrm{meV} / \mathrm{Mn})$ & Short Range Energy $(\mathrm{meV} / \mathrm{Mn})$ & Total Energy $(\mathrm{meV} / \mathrm{Mn})$ \\
\hline $\mathrm{CO}(\pi, \pi, 0), \mathrm{OO}$ 'zig-zag' & -33.2 & -278.4 & -311.6 \\
$\mathrm{CO}(\pi, \pi, \pi), \mathrm{OO}$ 'zig-zag' & -33.2 & -270.9 & -304.2 \\
$\mathrm{CO}(\pi, \pi, 0), \mathrm{OO}(\pi / 2, \pi / 2)$ & -65.0 & -218.0 & -283.0 \\
$\mathrm{CO}(\pi, \pi, \pi), \mathrm{OO}(\pi / 4,3 \pi / 4)$ & -15.9 & -290.8 & -306.7 \\
\hline
\end{tabular}

neighboring sites ${ }^{22}$ If applied to our 'zig-zag' orbital configuration, CE-type antiferromagnetism is found. Magnetic energy differences per site between CE-type antiferromagnetism and ferromagnetic ordering are $\sim 20 \mathrm{meV}$, therefore of the same order than the uniform strain energies. Yunoki and co-workers ${ }^{14}$ have shown how the magnetic interaction may help to stabilize $(\pi, \pi, 0)$ ordering relative to $(\pi, \pi, \pi)$ ordering; the energetics found in their work are sufficiently similar to those found by us that it is not clear which mechanism is dominant. Similarly, we have adopted a strictly classical, insulating model for the electronic degrees of freedom, but our electron-lattice coupling and lattice interaction could be easily carried over to an itinerant electron model (at least within the conventional adiabatic approximation for electron-lattice coupling). Use of such a model to explore strain effects on a second order weak amplitude ('Charge Density Wave' instability) electronic charge and orbital ordering transition would be very interesting. Finally, our calculation involves only relatively small length scales, and therefore has not included elastic compatibility and other long range effects. We hope our results may be useful as input parameters for calculations of longer length scale phenomena.

In summary, a classical model for charge and orbital ordering has been formulated and analyzed. The important new feature is that elastic energies as well as the cooperative Jahn-Teller coupling are included. Our main finding is that energetics associated with long ranged strain are at least as important as those from other (e.g. magnetic) sources in determining the ground state of the system. We find in particular that uniform strain is of vital importance in stabilizing the 'zig-zag chain' orbital ordering pattern observed in many manganite systems.

Acknowledgments. MJC acknowledges financial support from Churchill College (Cambridge, UK), AJM from the University of Maryland-Rutgers MRSEC (NSFDMR-00080008), and KHA from US DOE.
1 J.M.D. Coey, M. Viret and S. von Molnar, Adv. Phys. 48, 167 (1999); Colossal Magnetoresistance Oxides edited by Y. Tokura (Gordon \& Breach, Monographs in Condensed Matter Science, London, 1999); M.B. Salamon and M. Jaime, Rev. Mod. Phys. 73, 583 (2001); E. Dagotto, T. Hotta, and A. Moreo, Physics Reports 344, 1 (2001).

2 D.I. Khomskii and K.I. Kugel, Europhys. Lett. 55, 208 (2001).

3 K. Ahn and A. J. Millis, Phys. Rev. B 64115103 (2001).

4 E.B. Nyeanchi, I.P. Krylov, X.-M. Zhu and N. Jacobs, Europhys. Lett. 48, 228 (1999).

${ }^{5}$ C.S. Nelson et al, cond-mat/0303228 (unpublished).

${ }^{6}$ P. Levy et al, Phys. Rev. B 62, 6437 (2000).

7 V. Podzorov et al, Phys. Rev. B 64, 140406 (2001).

8 See, for instance, R.E. Reed-Hill \& R. Abbaschian, Physical Metallurgy Principles, 3rd ed. (PWS-Kent Publishing Company, Boston, 1991), pp. 561-587.

9 J.B. Goodenough, Phys. Rev. 100, 564 (1955).

10 J. Kanamori, J. Appl. Phys. 31, 14S (1960).

11 K.I. Kugel and D.I. Khomskii, Sov. Phys. JETP 37, 725 (1974).

12 S.K. Mishra, R. Pnadit and S. Satpathy, Phys. Rev. B 56, 2316 (1997); G. Jackeli, N.B. Perkins, N.M. Plakida, Phys. Rev. B 62, 372 (2000); S. Fratini, D. Feinberg and M. Grilli, The European Physical Journal B 22, 157 (2001)

13 A.J. Millis, Phys. Rev. B 53, 8434 (1996)
14 S. Yunoki, T. Hotta, and E. Dagotto, Phys. Rev. Lett. 84, 3714 (2000).

15 E. O. Wollan and W. C. Koehler, Phys. Rev. 100, 545 (1955).

16 S. Ishihara and S. Maekawa, Rep. Prog. Phys. 65, 561 (2002) and references therein.

17 K.H. Ahn, A.J. Millis, Phys. Rev. B 58, 3697 (1998);61, 13545 (2000).

18 M. J. Calderón, A.J. Millis and K. Ahn, to be published.

19 Note that, in reality, a whole single crystal cannot dramatically change its dimensions due to the internal strain but rather different domains where strain sets in different directions would be expected. Such martensitic domains are typically much larger than the sample-sizes numerically accessible to us, so we consider only single-domain behavior.

20 A. Urushibara et al, Phys. Rev. B 51, 14103 (1995) have estimated $C_{B}=143 \mathrm{GPa}$ for $\mathrm{La}_{0.83} \mathrm{Sr}_{0.17} \mathrm{MnO}_{3}$ from ultrasound experiments; R.D. King-Smith and D. Vanderbilt, Phys. Rev. B 49, 5828 (1994) calculated $C_{B}=150-200$ GPa for many different perovskites; A. Congeduti et al, Phys. Rev. B 63184410 (2001) give $C_{B}=175 \mathrm{GPa}$ for $\mathrm{La}_{0.75} \mathrm{Ca}_{0.25} \mathrm{MnO}_{3}$ from x-ray diffraction data.

21 R. Maezono, S. Ishihara, and N. Nagaosa, Phys. Rev. B 58, 11583 (1998).

22 A.J. Millis, Phys. Rev. B 55, 6405 (1997). 